\title{
E-nnovation: E-Business und Nachhaltigkeit
}

\author{
Bislang sind Umweltentlastungen durch Elec- \\ tronic Business nichtintendierte Nebeneffekte. \\ Der wirtschaftliche und ökologische Bedeu- \\ tungszuwachs des E-Business machen für die \\ Zukunft aber eine gezielte Berücksichtigung \\ von Umweltschutzanforderungen erforderlich. \\ Von Siegfried Behrendt und \\ Christine Henseling
}

m Rahmen des Forschungsvorhabens E-nnovation wurde erstmalig eine systematische Übersicht und Auswertung der möglichen Chancenpotenziale des E-Business für zukunftsfähige Produktnutzungssysteme erarbeitet. Zu diesen Potenzialen zählen beispielsweise lebenszyklusweite Ökoeffizienz, Service-Design, Dematerialisierung, Wieder- und Weiterverwendung, Recycling und nachhaltiges Wissensmanagement. Die Untersuchung hat ein heterogenes und fein unterteiltes Bild ergeben. So ist deutlich geworden, dass die teilweise hohen Erwartungen an eine Verringerung des Ressourcenverbrauchs und eine reduzierte Inanspruchnahme der Umwelt durch Internet und E-Business sich bisher nicht erfüllt haben. Durch die Digitalisierung von Gütern und Dienstleistungen erfolgt bislang weder automatisch eine spürbare Dematerialisierung von Produktion und Warenströmen, noch tragen digitale Produkte wie Internet-Zeitungen oder Musikdateien und Vertriebsformen zu einer gewichtslos werdenden Ökonomie bei. Vielmehr hängen die Umweltprofile von Anwendungen im EBusiness von einer Vielzahl von Faktoren und Rahmenbedingungen ab. Dennoch bestehen beachtenswerte Potenziale zur Unterstützung nachhaltiger Produktnutzungssysteme im E-Business. Grundsätzlich können die folgenden Strategiefelder unterschieden werden.

\section{Strategiefelder im E-Business}

Ökologische Gestaltung der Netzinfrastruktur: Verschiedene Ökobilanzen illustrieren, dass die ökologische Nettobilanz von Anwendungen in der Informations- und Kommunikationstechnologie (IKT) entscheidend auch von ökologischen Eigenschaften der Hardware und der Infrastruktur, wie dem Energieverbrauch für die Herstellung und Nutzung, beeinflusst wird.

Dematerialisierung durch digitale Medienprodukte: Gemeint ist, dass materielle Produkte durch digitalisierte Güter ersetzt werden, man könnte sagen „Kilobyte statt Kilogramm“. Die Umweltwirkungen hängen hierbei maßgeblich von den Rahmenbedingungen der Nutzung ab. So sind elektronische Medien oftmals kein Substitut für Print- oder andere Medien, sondern eine Ergänzung, was den Umweltverbrauch tendenziell erhöht.

Ressourcenproduktivität in der Leistungserstellung: Die Verbreitung von E-Business in Beschaffung und Vertrieb ist vor allem in den Business-to-Business-Beziehungen (B2B) seit Jahren in vollem Gange und wird sich voraussichtlich in Zukunft weiter erhöhen. Dies gilt insbesondere für ein elektronisch gestütztes Supply Chain Management. Die vorliegenden Daten weisen auf ein relevantes Verbesserungspotenzial bei der Ressourcenproduktivität hin. Gleichwohl lässt sich kein ökologischer Quantensprung, wie er insbesondere in der US-amerikanischen Literatur teilweise angenommen wird, erkennen (Cohen 1999).

Kundeninformation und Kundenintegration: Das Internet bietet Möglichkeiten zur Erhöhung der Markttransparenz. Kunden können sich über die Angebotsvielfalt besser denn je informieren. Methoden des One-to-one- oder Database-Marketing zielen auf eine frühzeitige individuelle Kundenansprache und lang-
Abbildung 1: Strategiefelder für eine nachhaltige Produktnutzung im E-Business

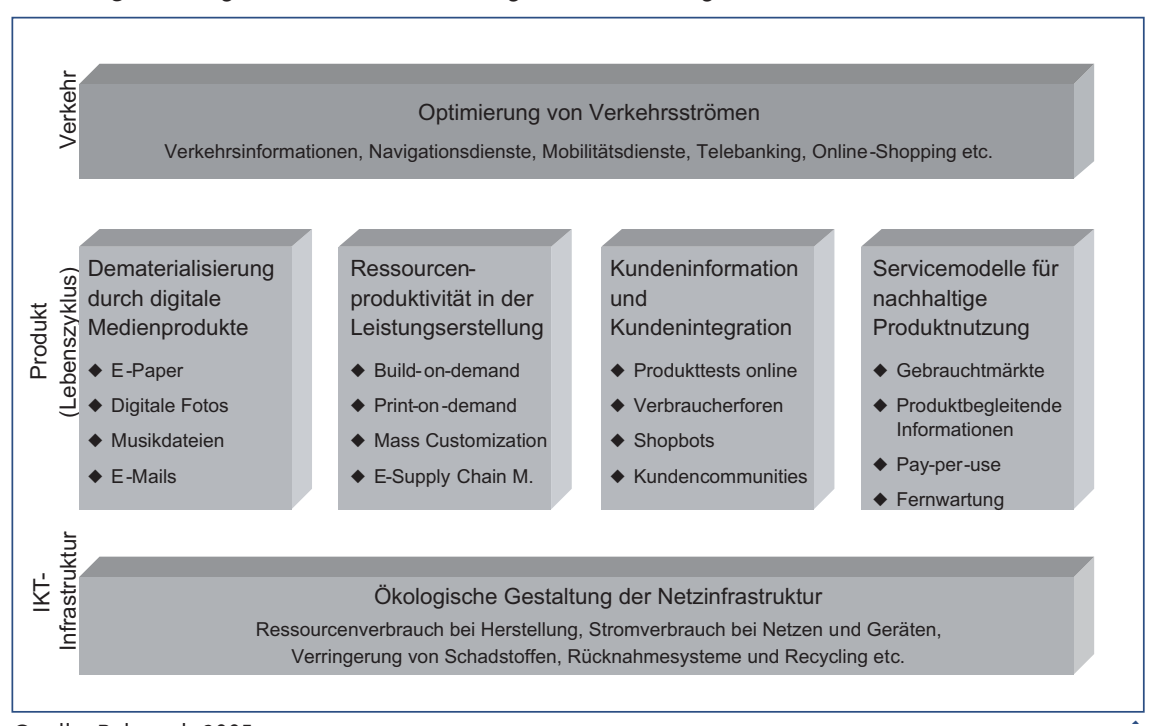

Quelle: Behrendt 2005 


\section{„Wie eine erste ökobilanzielle Abschätzung zeigt, führen E-Paper-Lösungen nicht automatisch zur Verringerung des Energie- und Umweltverbrauchs, sondern können diesen sogar erhöhen."}

fristige Kundenbindung. Erwartet wird, dass dadurch auch ökologische Produkt- und Dienstleistungsinnovationen und die Ausbildung ökologischer Märkte erleichtert werden. Auf der Risikoseite stehen die zunehmende Datenflut und die mediale Reizüberflutung.

Servicemodelle für nachhaltige Produktnutzung: Ein weiterer bedeutsamer Strategieansatz für nachhaltige E-Business-Lösungen liegt in innovativen Dienstleistungsmodellen. Das Spektrum reicht von elektronischen Gebrauchtmärkten, produktbegleitenden Informationssystemen, internetbasierter Fernablese, -wartung und -reparatur bis hin zu Pay-per-Use-Konzepten, die durch Smart Label erst praktikabel werden.

Optimierung von Verkehrsströmen im Sinne einer virtuellen Mobilität: Die größten verkehrsentlastenden Potenziale durch die Nutzung von IKT bestehen im Bereich des Geschäftsverkehrs bei Telearbeit und virtuellen Meetings. In anderen Anwendungsfeldern, wie Videokonferenzen, Online-Angeboten im Tourismus, Teleshopping oder Telebanking sind Verkehrsentlastungspotenzielle bisher eher gering. Mit verkehrsinduzierenden Effekten ist bei Chats und interaktiv vernetzten Spielwelten, sogenannten Multi User Dungeons zu rechnen. Unbekannt sind die Auswirkungen von Call-Center-Lösungen, Fernwartung, E-Learning und E-Teaching.

Im Folgenden sollen vier Felder näher vorgestellt werden, die sich mit Blick auf die Unterstützung nachhaltiger Produktnutzungssysteme als besonders interessant herausgestellt haben.

\section{eBay und Co. - Gebrauchtgütermärkte im Internet und ihre ökologischen Effekte}

Chancen zur Erschließung bisher nicht genutzter Umweltentlastungspotenziale bestehen bei der Weiterentwicklung internetgestützter Gebrauchtmärkte. Dieses Potenzial beruht im Wesentlichen auf der Chance, durch die Vermarktung gebrauchter Güter die Lebens- und Nutzungsphase von Produkten zu verlängern und so zusätzliche Umweltbelastungen durch Neuanschaffungen zu vermeiden. Das Internet vereinfacht die Suche, Anbahnung und Abwicklung des Gebrauchtwarenhandels. Es senkt damit die Transaktionskosten und schafft hier einen Quantensprung im Gebrauchtwarenhandel. Allerdings stellt sich in diesem Zusammenhang die Frage nach Reboundeffekten. Bislang gibt es keine Untersuchung über die Umwelt- und Verkehrseffekte von Online-Gebrauchtgütermärkten. Für die Abschätzung der potenziellen Substitution von Einkaufsfahrten und Neukäufen durch den Onlinehandel mit Gebrauchtgütern sind Erkenntnisse hinsichtlich des Einkaufs- und Mobilitätsverhaltens der Konsumenten und über dessen Veränderung durch E-Commerce notwendig. Von besonderem Interesse ist unter anderem die Frage, ob der Kauf gebrauchter Produkte sonstige Produktkäufe substituiert oder ob dies zu einem additiven Konsum führt. Ein wesentlicher Einflussfaktor auf die ökologische Bilanz sind die Transportaufwendungen. Wichtig wäre hier zu untersuchen, über welche Entfernungen die Produkte gehandelt werden, ob es, in Bezug auf die Transportentfernung, Unterschiede zwischen verschiedenen Produktgruppen gibt und welche Veränderungen sich bei den Auslieferungsverkehren durch eine $\mathrm{Zu}$ nahme von Einzelsendungen ergeben. Ein weiterer wichtiger Faktor, der für die ökologischen Effekte von online gehandelten Gebrauchtgütern eine Rolle spielt, sind die Verpackungsmaterialien, die bei der Einzelversendung der Produkte anfallen.

\section{Ressourcenproduktivität durch produktbe- gleitende Informationssysteme}

Smart-Label-basierte Lösungen bieten grundsätzlich die Möglichkeit, sämtliche Produktlebensphasen vom Design bis zur Entsorgung des Produktes zu erfassen. Im B2B-Bereich lassen sich mithilfe von Smart Label Kosten in verschiedenen Bereichen senken. Dazu gehören Logistik, Service, Wartung, Reparatur, Garantieforderungen, Weiterverwendung und Entsorgung von Produkten. Im Business-to-(B2C)-Consumer Bereich sind die Anwendungspotenziale weitaus spekulativer. $\mathrm{Ne}$ ben aussichtsreichen Anwendungen wie der präventiven Fernwartung oder der Überwachung der Lebensmittelkette lassen viele Anwendungen in Konsumgüterbereichen nur schwer einen Zusatznutzen für den Verbraucher erkennen. Beispielhaft ist der smarte Kühlschrank, der automatisch Waren nachbestellt.

Fragen des individuellen Kundennutzens beziehungsweise der Akzeptanz sind bei dieser technologiegetriebenen Entwicklung weitgehend ungeklärt. Als große Akzeptanzhürden dürften sich die informationelle Überforderung sowie Aspekte der Privatheit und des Datenschutzes erweisen. Mit Blick auf das Marktpotenzial sind daher eher Nischenanwendungen wahrscheinlich. Insbesondere die Vorstellung, anstelle von Sachgütern nur deren Nutzung, also Dienstleistungen zu verkaufen („Pay-per-Use“) wird durch Smart Label in vielen Fällen erst praktikabel. Dies berührt aber nicht die viel wichtigeren psychologischen und sozialen Funktionen des Produktkaufs, sodass kaum davon ausgegangen werden kann, dass Smart Labels die Bereitschaft zum pay-per-use, über die bereits ohnehin erschlossenen Bereiche des Nutzungskaufs hinaus, wesentlich fördern wird.

Inzwischen gibt es mehrere Prototypen für Foliendisplays, die als Alternativen zu konventionellen Röhren- und Flachbildschirmen, aber auch zu Papier, entwickelt wurden. Neben noch zu lösenden technologischen Herausforderungen und der Fra- 
Abbildung 2: Ergebnisse der Berechnung der kumulierten Energieaufwendungen der drei Zeitungsvarianten

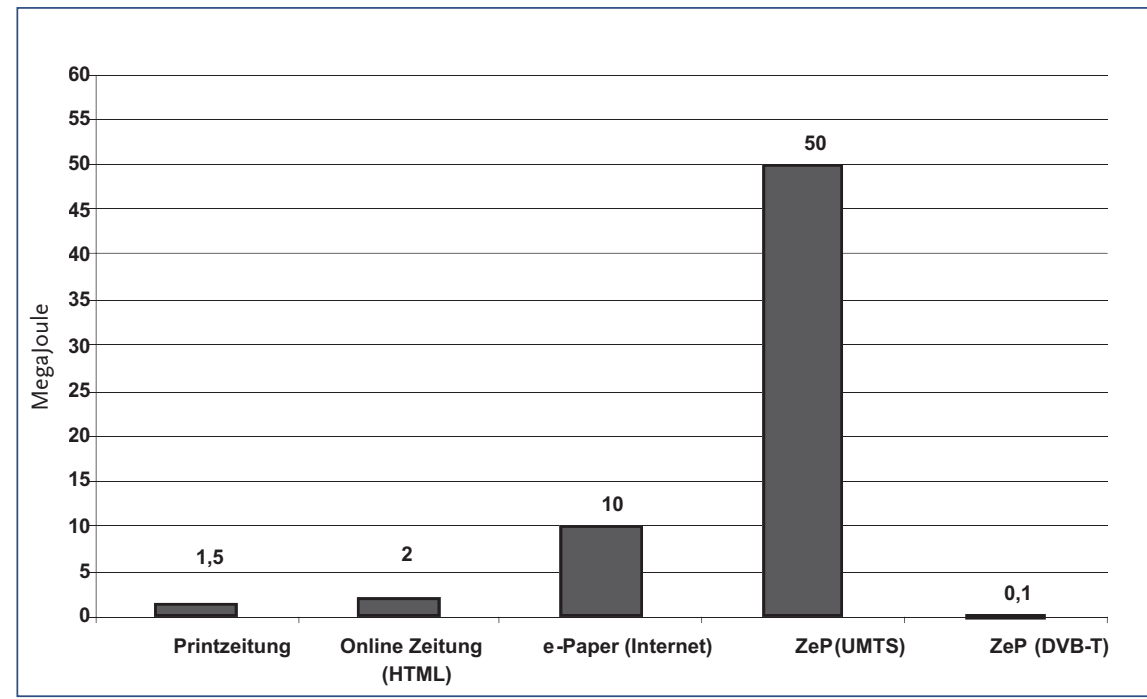

Quelle: Kamburow, 2004

ge nach frühen Marktkonzepten hängt die erfolgreiche Weiterentwicklung und Nutzung der Technologie von der Integration einer Reihe von Nachhaltigkeitsaspekten ab. Wie eine erste ökobilanzielle Abschätzung des Instituts für Zukunftsstudien und Technologiebewertung (IZT) zeigt, führen E-Paper-Lösungen nicht automatisch zur Verringerung des Energie- und Umweltverbrauchs, sondern können diesen sogar erhöhen. Im Rahmen einer orientierenden Ökobilanz wurde anhand der Methode des kumulierten Primärenergieaufwandes eine Abschätzung des Energieverbrauchs der Zeitung auf elektronischem Papier durchgeführt und mit herkömmlichen Print- und Online-Zeitungen verglichen. Die Resultate sind in Abbildung 2 dargestellt.

\section{Dematerialisierung durch Zeitung auf elektronischem Papier?}

Es wird deutlich, dass das Lesen der Zeitung auf elektronischem Papier den mit Abstand höchsten Primärenergieaufwand benötigt und dabei der Energieaufwand der mobilen Variante (Datenübertragung über das UMTS-Mobilfunknetz) noch einmal deutlich über dem Energieaufwand für die teilmobile Lösung (Datenübertragung und Herunterladen über Internet und PC) liegt. Dies ist bei der mobilen Variante in erster Linie auf den hohen Primärenergieaufwand für die Datenübertragung über das UMTS-Mobilfunknetz zurückzuführen. Da zudem die gesamte Zeitungsausgabe und nicht nur einige Artikel, wie bei der Variante Online-Zeitung, übertragen werden, ist auch die übertragene Datenmenge ungleich größer als bei der OnlineZeitung.

Die elektronische Zeitungstechnologie weist mit einem niedrigen Energieaufwand für Herstellung und dem Gebrauch eines Foliendisplays, Materialeffizienz und Wiederverwendbarkeit verschiedene günstige Umwelteigenschaften auf. Entgegen frü- heren Erwartungen zeigt die ökologische Grobabschätzung, dass sich diese günstigen Eigenschaften nicht automatisch in einer Umweltentlastung niederschlagen. Vielmehr hängt die Nettobilanz von den Einflussfaktoren Nutzungsintensität, Endgeräte und dem Ausdruck von Nachrichten im Anwendungssystem ab.

Mit Abstand der wichtigste Parameter ist der durch die elektronischen Übertragungswege verursachte Energieaufwand. Speziell das Beispiel einer Datenübertragung von Inhalten über das UMTS-Mobilfunksystem zeigt, dass einerseits Foliendisplays aufgrund ihrer Eigenschaften für mobile Anwendungen prädestiniert sind und mit großer Wahrscheinlichkeit hier auch ihr Hauptanwendungsgebiet finden werden. Aber andererseits werden genau diese mobilen Dienste, durch die hohen Energieaufwendungen einer quasi überall gegebenen Verfügbarkeit, die ökologischen Vorteile einer elektronischen Zeitung überkompensieren. Daher ist der Blick auf alternative Übertragungswege zu richten. Insbesondere DAB (Digital Audio Broadcasting) und DVB-T (Digital Video Broadcasting Terrestrial), die deutlich weniger Energie für das Versenden der Zeitung benötigen, erscheinen hier interessant.

\section{Empowerment von Verbrauchern durch internetgestützte Information}

Das Internet bietet vielversprechende Möglichkeiten der Verbesserung der Verbraucherinformation zu ökologischen und sozialen Produkteigenschaften sowie der Vernetzung von Akteuren und der Etablierung neuer Anbieter. Sollen nachhaltige Produkte durch breite Käuferschichten bevorzugt werden, bedarf es ausreichender und glaubwürdiger Informationen über Qualität, Funktionalität, Preis und ökologische Eigenschaften der Produkte. Die durch Interaktivität und Individualisierungspotenziale des Internets ermöglichte zielgruppenspezifische Massenkommunikation lässt eine umfangreiche, kostengünstige und gleichzeitig auf die Informationsinteressen der jeweiligen Zielgruppen zugeschnittene Verbraucherinformation zu.

Die Bandbreite solcher Angebote im Internet reicht von etablierten Einrichtungen des Verbraucherschutzes bis hin zu neuen Dienstleistern, die Internetportale, Online-Einkaufsführer für Produkte und Dienstleistungen oder Verbraucherforen betreiben. Eine Analyse von Verbraucherinformationen im Internet ergab, dass diese neuen Intermediäre, hier insbesondere die Verbraucherforen und Preisvergleiche, die größten Nutzerzahlen erreichen und die innovativen Features des Internet am meisten nutzen. Allerdings spielen bei diesen Anbietern umweltrelevante Informationen bisher nur eine sehr geringe Rolle. Hier stellt sich aus Sicht einer nachhaltigen Produktnut- $\rightarrow$ 
zung die Frage, wie die Anbieter für solche Aspekte sensibilisiert werden können.

Auf der anderen Seite stehen die etablierten Einrichtungen des Verbraucherschutzes und der vergleichenden Warentests, die bereits nachhaltigkeitsrelevante Informationen liefern. Diese erreichen in der Regel eine weitaus kleinere Nutzerzahl. Des Weiteren wird von ihnen das Internet häufig primär als Informationsund Vertriebsmedium genutzt, während andere Eigenschaften wie die Möglichkeiten zur Individualisierung von Informationen oder interaktive Elemente und Community-Angebote noch wenig eingesetzt werden. Nutzer, die Produktinformationen im Internet suchen, erwarten aber nicht nur, die Print-Inhalte aufbereitet vorzufinden, sondern sie erwarten von einem Online-Angebot weitere Servicefunktionen. In diesem Sinne sollte geprüft werden, wie die Anbieter ökologischer Informationen die innovativen Eigenschaften des Internets stärker nutzen und in ihre Angebote einbeziehen können.

\section{Fazit}

Die Chancen bestimmter E-Business-Anwendungen werden nicht durch das Vorhandensein der bloßen technischen Möglichkeit erschlossen. Zu ihrer Erschließung kommt es auf die gezielte Berücksichtigung zentraler Einflussfaktoren an. Eine frühzeitige Auseinandersetzung mit diesen Aspekten hilft Unsicherheiten bei Technologieentwicklung, Markteinführung und Geschäfts- und Erlösmodellen zu minimieren, die ökologische Richtungssicherheit zu erhöhen und ist letztlich ein Erfolgsfaktor bei der Einführung dieser neuen Technologie.

\section{Literatur}

Behrendt, S. Dematerialisierung durch E-Paper? IZT WerkstattBericht Nr. 66. Berlin 2004

Behrendt, S.: Produktbegleitende Informationssysteme auf der Basis von

Smart Label. IZT Arbeitsbericht Nr. 7. Berlin 2004.

Behrendt, S. / Henseling, C. / Fichter, K. / Bierter, W.: Chancenpotenziale für nachhaltige Produktnutzungssysteme im E-Business. IZT WerkstattBericht Nr. 71. Berlin 2005

Cohen, M. / Neven, M.: Greening the Internet. Ten Ways E-Commerce could affect the Environment and what we can do. In: IMP-Magazine, 1999, www.cosp.org/imp/october_99/10_99Cohen.htm.

Fichter, K.: Modelle der Nutzerintegration in den Innovationsprozess, Möglichkeiten und Grenzen der Integration von Verbrauchern in Innovations prozesse für nachhaltige Produkte und Produktnutzungen in der Internetökonomie. IZT WerkstattBericht Nr. 75. Berlin 2005.

Henseling, C. / Fichter, K.: Online-Marktplätze für Gebrauchtgüter. IZT Arbeitsbericht Nr. 8. Berlin 2004.

Henseling, C. / Fichter, K.: Produktinformationen für Verbraucher im Internet. IZT Arbeitsbericht Nr. 16. Berlin 2005.

Kamburow, C.: E-Paper - Erste Abschätzung der Umweltauswirkungen. Eine ökobilanzielle Betrachtung am Beispiel des Nachrichtenmediums Zeitung. IZT WerkstattBericht Nr. 67. Berlin 2004.

\section{AUTOREN + KONTAKT}

Siegfried Behrendt ist Projektleiter am Institut für Zukunftsstudien und Technologiebewertung sowie Lehrbeauftragter an der Technischen Fachhochschule und der Fachhochschule für Wirtschaft in Berlin.

Institut für Zukunftsstudien und Technologiebewertung IZT, Schopenhauer Str. 26, 14129 Berlin. E-Mail: s.behrendt@izt.de

Christine Henseling ist wissenschaftliche Mitarbeiterin am Institut für Zukunftsstudien und Technologiebewertung Institut für Zukunftsstudien und Technologiebewertung
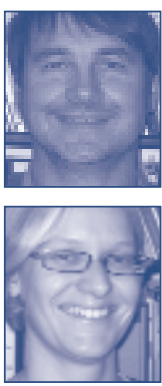

IZT, Schopenhauer Str. 26, 14129 Berlin. E-Mail: c.henseling@izt.de
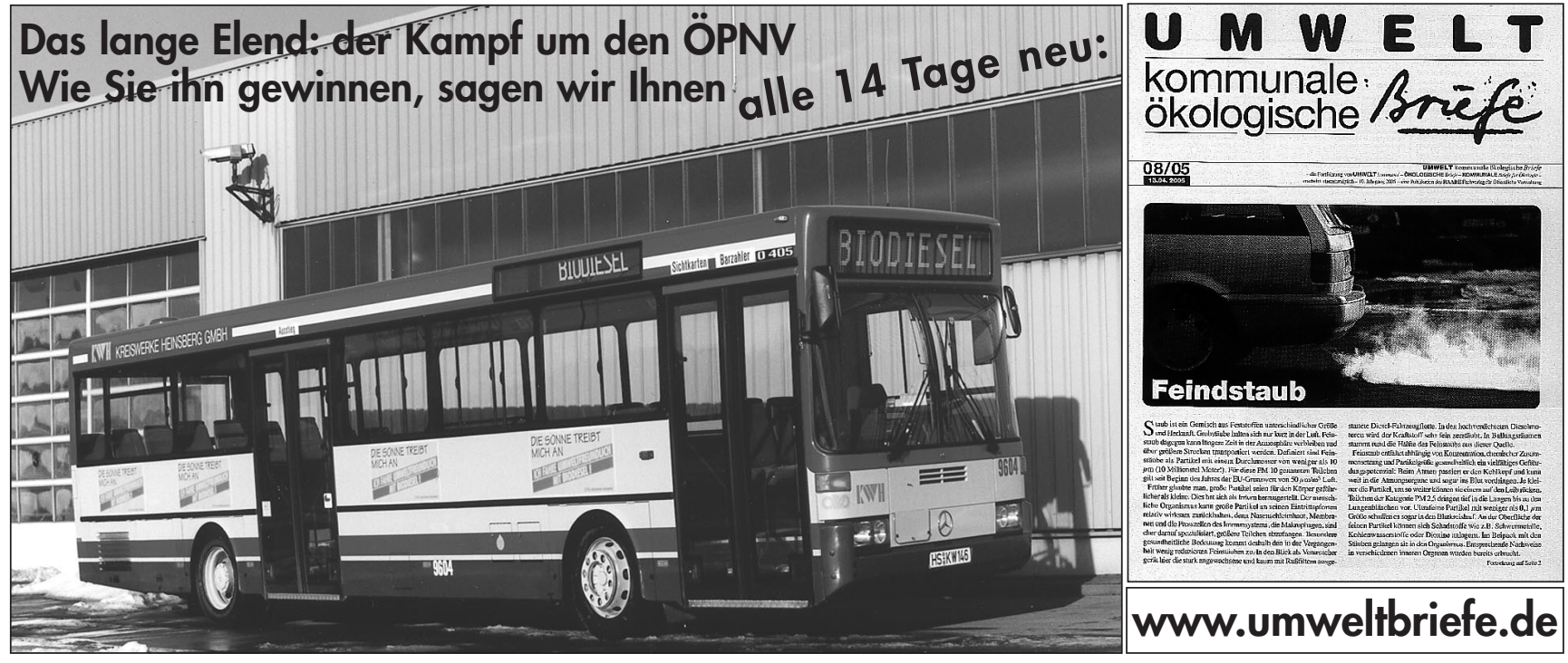
(c) 20I0 Authors; licensee IÖW and oekom verlag. This is an article distributed under the terms of the Creative Commons Attribution Non-Commercial No Derivates License (http://creativecommons.org/licenses/by-nc-nd/3.o/), which permits unrestricted use, distribution, and reproduction in any medium, provided the original work is properly cited. 